12th International Symposium on Cosmology and

Particle Astrophysics (CosPA 2015)

International Journal of Modern Physics: Conference Series

Vol. 43 (2016) 1660197 (13 pages)

(C) The Author(s)

DOI: $10.1142 / \mathrm{S} 2010194516601976$

\title{
Theory for Neutrino Mixing
}

\author{
Xiao-Gang He \\ INPAC, SKLPPC, and Department of Physics, \\ Shanghai Jiao Tong University, Shanghai 200240, China \\ National Center for Theoretical Sciences and \\ Physics Department of National Tsing Hua University, \\ Hsinchu 300, Taiwan \\ CTS, CASTS, and Physics Department, \\ National Taiwan University, Taipei 106, Taiwan \\ hexg@phys.ntu.edu.tw
}

Published 7 July 2016

\begin{abstract}
Since the discovery of neutrino oscillations, for which Takaaki Kajita and Arthur B. McDonald were awarded the 2015 Nobel prize in physics, tremendous progresses have been made in measuring the mixing angles which determine the oscillation pattern. A lot of theoretical efforts have been made to understand how neutrinos mix with each other. Present data show that in the standard parameterization of the mixing matrix, $\theta_{23}$ is close to $\pi / 4$ and the $\mathrm{CP}$ violating phase is close to $-\pi / 2$. In this talk I report results obtained in arXiv:1505.01932 (Phys. Lett. B750(2015)620) and arXive:1404.01560 (Chin. J. Phys.53(2015)100101) and discuss some implications for theoretical model buildings for such mixing pattern. Specific examples for neutrino mixing based on $A_{4}$ family symmetry are given.
\end{abstract}

Keywords: neutrino; oscillation; mixing; CP violation.

\section{Introduction}

The 2015 Nobel prize in physics was awarded to Takaaki Kajita and Arthur B. McDonald for the discovery of neutrino oscillations. ${ }^{1,2}$ Neutrino oscillations imply that neutrinos have mass and mix with each other. The mixing matrix is defined by the interaction of $W$ boson with neutrinos and charged leptons as the following,

$$
L=-\frac{g}{\sqrt{2}} \bar{E} \gamma^{\mu} V_{P M N S} L \nu W_{\mu}^{-}+H . C .
$$

where $L=\left(1-\gamma_{5}\right) / 2 . E_{L}$ and $\nu_{L}$ indicate the charged leptons $E=(e, \mu, \tau)$ and neutrinos $\nu=\left(\begin{array}{lll}\nu_{1} & \nu_{2}, \nu_{3}\end{array}\right) . V_{P M N S}$ is the Pontecorvo-Maki-Nagakawa-Sakata

This is an Open Access article published by World Scientific Publishing Company. It is distributed under the terms of the Creative Commons Attribution 4.0 (CC-BY) License. Further distribution of this work is permitted, provided the original work is properly cited. 
mixing matrix ${ }^{3}$ which is a $3 \times 3$ unitary matrix,

$$
V_{P M N S}=\left(\begin{array}{ccc}
V_{e 1} & V_{e 2} & V_{e 3} \\
V_{\mu 1} & V_{\mu 2} & V_{\mu 3} \\
V_{\tau 1} & V_{\tau 2} & V_{\tau 3}
\end{array}\right)
$$

and in the standard parametrization used by the Particle Data Group ${ }^{4,5}$ is of the following form

$$
V_{P M N S}=\left(\begin{array}{ccc}
c_{12} c_{13} & s_{12} c_{13} & s_{13} e^{-i \delta} \\
-s_{12} c_{23}-c_{12} s_{23} s_{13} e^{i \delta} & c_{12} c_{23}-s_{12} s_{23} s_{13} e^{i \delta} & s_{23} c_{13} \\
s_{12} s_{23}-c_{12} c_{23} s_{13} e^{i \delta} & -c_{12} s_{23}-s_{12} c_{23} s_{13} e^{i \delta} & c_{23} c_{13}
\end{array}\right),
$$

where $c_{i j}$ and $s_{i j}$ are $\cos \theta_{i j}$ and $\sin \theta_{i j}$, respectively. If neutrinos are Majorana particles, there are also two CP violating Majorana phases $\alpha_{i}$. In that case one should multiply an additional diagonal matrix $P=\operatorname{diag}\left(e^{i \alpha_{1} / 2}, e^{i \alpha_{2} / 2}, 1\right)$ on the right in the above.

Neutrino oscillations are caused by the missing match between weak and mass eigen-state bases. Neutrinos are produced by weak interactions. At production point they are in weak interaction eigen-state $\left|\nu_{\alpha=e, \mu, \tau}\right\rangle$. It is a mixture of mass eigenstate $\left|\nu_{i=1,2,3}\right\rangle$ with $\left|\nu_{\alpha}\right\rangle=\sum_{i} V_{\alpha i}\left|\nu_{i}\right\rangle$. After traveling a distance $L$ in free space along a given direction, the state becomes

$$
\left|\nu_{\alpha}(L)>=\sum_{i} V_{\alpha i} e^{i\left(p_{i} L-E t\right)}\right| \nu_{i}>.
$$

When a measurement of spice $\nu_{\beta}$ is applied at distance $L$, the probability amplitude of finding $\nu_{\beta}$ is given by

$$
A\left(\nu_{\alpha} \rightarrow \nu_{\beta}\right)=<\nu_{\beta} \mid \nu_{\alpha}(L)>=\sum_{i} V_{\beta i}^{*} V_{\alpha i} e^{i\left(p_{i} L-E t\right)} .
$$

In the case where neutrino masses are much smaller then the energy scale $E$, to a good approximation $t \approx L$ and $p_{i}=\sqrt{E^{2}-m_{i}^{2}} \approx E-m_{i}^{2} / 2 E$. With these approximations, the probability $P\left(\nu_{\alpha} \rightarrow \nu_{\beta}\right)$ for $\nu_{\alpha} \rightarrow \nu_{\beta}$ transition is given by

$$
\begin{aligned}
P\left(\nu_{\alpha} \rightarrow \nu_{\beta}\right) & =\left|\sum_{i} V_{\beta i}^{*} V_{\alpha i} e^{-i m_{i}^{2} L / 2 E}\right|^{2} \\
& =\delta_{\alpha \beta}-4 \sum_{i>j} \operatorname{Re}\left(V_{\alpha i}^{*} V_{\beta i} V_{\alpha j} V_{\beta j}^{*}\right) \sin ^{2}\left(\Delta m_{i j}^{2} L / 4 E\right) \\
& -2 \sum_{i>j} \operatorname{Im}\left(V_{\alpha i}^{*} V_{\beta i} V_{\alpha j} V_{\beta j}^{*}\right) \sin \left(\Delta m_{i j}^{2} L / 2 E\right),
\end{aligned}
$$

where $\Delta m_{i j}^{2}=m_{i}^{2}-m_{j}^{2}$. It is convenient to write $\sin ^{2}\left(\Delta m_{i j}^{2} L / 4 E\right)=$ $\sin ^{2}\left(1.267\left(\Delta m_{i j}^{2} / \mathrm{eV}^{2}\right)(L / K m)(G e V / E)\right)$.

The above equation clearly shows that a non-zero probability for a $\nu_{\alpha}$ neutrino oscillates into another type $\nu_{\beta}$ requires at least one of the $\Delta m_{i j}^{2}$ be non-zero and the mixing elements $V_{\alpha i}$ cannot just be $\delta_{\alpha i}$. Measurements of neutrino oscillations 
may obtain information about $\Delta m_{i j}^{2}$ and the mixing angles $\theta_{i j}$ and also CP violating phase $\delta$. Note that the Majorana phases do not show up anywhere in the oscillation probability and therefore no information can be obtained from oscillation experiments.

Tremendous experimental progresses have been made in obtaining information about the neutrino mixing parameters. ${ }^{4}$ Early studies of neutrino mixing patterns range from small mixing angles ${ }^{6}$ similar to quark mixing pattern to bi-maximal mixing, ${ }^{7}$ and to the famous tribi-maximal mixing. ${ }^{8}$ The mixing pattern with a small $\theta_{12}$ was ruled out by large mixing from solar neutrino oscillation data, ${ }^{1,4}$ and that with a small $\theta_{23}$ was ruled out by the atmospherical neutrino oscillation data $^{2,4}$ with $\theta_{23}$ is close to $\pi / 4$. The measurement of a non-zero $\theta_{13}$ from the Daya Bay $^{9}$ and Reno ${ }^{10}$ data started new direction for neutrino mixing study and also opened the possibility to have $\mathrm{CP}$ violation in the neutrino mixing matrix. Recent neutrino oscillation data from $\mathrm{T}_{2} \mathrm{~K}^{11}$ and $\mathrm{NO} \nu \mathrm{E}^{12}$ indicate that the $\mathrm{CP}$ violating phase in the mixing matrix is close to $-\pi / 2$. The new challenge for theoretical studies is then to understand why the neutrino mixing pattern has a $\theta_{23}=\pi / 4$ and $\delta=-\pi / 2$. There are many discussions about implications of $|\delta|=\pi / 2$ and $\theta_{23}=\pi / 4$. One of the commonly mentioned property for this type of mixing is the so called maximal CP violation because $|\delta|$ is $\pi / 2$. This is, strictly speaking, an incorrect statement because that the value of the Dirac phase is parametrization dependent. For example, even the absolute value of the Dirac phase is $\pi / 2$ in the standard parametrization, in the original Kobayashi-Maskawa parametrization for quarks $^{13}$ it is not $\pi / 2$ anymore. However, the special values for some of the mixing angles and the Dirac phase can still provide important information about neutrino mass matrix and can guide theoretical model buildings to search for the underlying theory.

\section{Neutrino mixing with $\theta_{23}=\pi / 4$ and $\delta=-\pi / 2$}

To have some model independent understanding of the mixing pattern with $\theta_{23}=$ $\pi / 4$ and $\delta=-\pi / 2$, let us reconstruct the neutrino mass matrix assuming that neutrinos are Majorana particles. In the basis where the charged lepton mass matrix is already diagonalized, the neutrino mass matrix defined by the term giving neutrino masses in the Lagrangian $(1 / 2) \bar{\nu}_{L} m_{\nu} \nu_{L}^{c}$, has the following form

$$
m_{\nu}=V_{P M N S} \hat{m}_{\nu} V_{P M N S}^{T},
$$

where $\hat{m}_{\nu}=\operatorname{diag}\left(m_{1}, m_{2}, m_{3}\right)$ with $m_{i}=\left|m_{i}\right| \exp \left(i \alpha_{i}\right)$. Here we have put Majorana phase information in the neutrino masses.

With $\delta=-\pi / 2$ and $\theta_{23}=\pi / 4, m_{\nu}$ has the following form ${ }^{14,15}$

$$
m_{\nu}=\left(\begin{array}{ccc}
a & c+i \beta & -(c-i \beta) \\
c+i \beta & d+i \gamma & b \\
-(c-i \beta) & b & d-i \gamma
\end{array}\right)
$$


where

$$
\begin{aligned}
& a=m_{1} c_{12}^{2} c_{13}^{2}+m_{2} s_{12}^{2} c_{13}^{2}-m_{3} s_{13}^{2} \\
& b=-\frac{1}{2}\left(m_{1}\left(s_{12}^{2}+c_{12}^{2} s_{13}^{2}\right)+m_{2}\left(c_{12}^{2}+s_{12}^{2} s_{13}^{2}\right)-m_{3} c_{13}^{2}\right) \\
& c=-\frac{1}{\sqrt{2}}\left(m_{1}-m_{2}\right) s_{12} c_{12} c_{13} \\
& d=\frac{1}{2}\left(m_{1}\left(s_{12}^{2}-c_{12}^{2} s_{13}^{2}\right)+m_{2}\left(c_{12}^{2}-s_{12}^{2} s_{13}^{2}\right)+m_{3} c_{13}^{2}\right) \\
& \beta=\frac{1}{\sqrt{2}} s_{13} c_{13}\left(m_{1} c_{12}^{2}+m_{2} s_{12}^{2}+m_{3}\right) \\
& \gamma=-\left(m_{1}-m_{2}\right) s_{12} c_{12} s_{13} .
\end{aligned}
$$

Note that in the most general case, because non-zero Majorana phases, the parameters $a, b, c, d, \beta$ and $\gamma$ are all complex.

The above matrix has a high level regularity pattern implying some underlying symmetry may be at work to produce it. Searching an underlying theory guided by symmetry principle may achieve this. Before doing this, however, it is worthwhile to understand more about the mass matrix in eq.(8). An immediate question one may ask is that if, in general, the neutrino mass matrix in eq.(8) always predicts $\delta=-\pi / 2$ and $\theta_{23}=\pi / 4$. The answer is negative. If $\delta=\pi / 2$ and $\theta_{23}=\pi / 4$, the neutrino mass matrix is given in a similar form as that in eq.(8), but $\beta$ and $\gamma$ need to be multiplied by a "-" sign. Therefore without further information given, a general mass matrix in the form given by eq.(8) can give $\delta= \pm \pi / 2$ and $\theta_{23}=\pi / 4$. Whether they predict $+\pi / 2$ or $-\pi / 2$, additional information need to be provided. Moreover, If neutrinos have Majorana phases, the general form does not imply that $\delta$ and $\theta_{23}$ must take $\pm \pi / 2$ and $\pi / 4$, respectively, neither. This can be understood by studying the following quantity

$$
m_{\nu} m_{\nu}^{\dagger}=V_{P M N S} \hat{m}_{\nu} \hat{m}_{\nu}^{\dagger} V_{P M N S}^{\dagger}
$$

The general form for neutrino mass in eq.(8) will give the " 12 " and " 13 " entries $A_{12,13}$ of $m_{\nu} m_{\nu}^{\dagger}$ as

$$
\begin{aligned}
A_{12}+A_{13}= & -i 2\left(a \beta^{*}+c \gamma^{*}-\beta d^{*}-\beta b^{*}\right) \\
= & -\left(\left|m_{1}\right|^{2}-\left|m_{2}\right|^{2}\right) s_{12} c_{12} c_{13}\left(c_{23}-s_{23}\right) \\
& -\left(\left|m_{1}\right|^{2} c_{12}^{2}+\left|m_{2}\right|^{2} s_{12}^{2}-\left|m_{3}\right|^{2}\right) s_{13} c_{13}\left(c_{23}+s_{23}\right) e^{-i \delta} \\
A_{12}-A_{13}= & 2\left(a c^{*}+c d^{*}-c b^{*}+\beta \gamma^{*}\right) \\
= & -\left(\left|m_{1}\right|^{2}-\left|m_{2}\right|^{2}\right) s_{12} c_{12} c_{13}\left(c_{23}+s_{23}\right) \\
& +\left(\left|m_{1}\right|^{2} c_{12}^{2}+\left|m_{2}\right|^{2} s_{12}^{2}-\left|m_{3}\right|^{2}\right) s_{13} c_{13}\left(c_{23}-s_{23}\right) e^{-i \delta}
\end{aligned}
$$

If the parameters in the set $P:\{a, b, c, \beta, \gamma\}$, are complex, the above equations can find solutions for other values of $\theta_{23}$ and $\delta$. Therefore the general neutrino mass matrix form does not imply that $\delta$ and $\theta_{23}$ must be $\pm \pi / 2$ and $\pi / 4$. If, however, the parameters in the set $P$ are all real, as long as $\sin \delta \neq 0$, one must have $s_{23}=c_{23}$ and 
$\delta= \pm \pi / 2$ as can be seen from the above two equations. From eq.(9) and eq.(11), one also finds that all eigen-masses $m_{i}$ are real (the Majorana phases are zero or $\pi)$. In this case the neutrino mass matrix can be rewritten as

$$
m_{\nu}=\left(\begin{array}{ccc}
A & C & -C^{*} \\
C & D^{*} & B \\
-C^{*} & B & D
\end{array}\right)
$$

with $A=a, B=b, C=c+i \beta$, and $D=d-i \gamma$. The most general $m_{\nu}$ can be written as ${ }^{14,15}$

$$
m_{\nu}=\left(\begin{array}{ccc}
e^{i p_{1}} & 0 & 0 \\
0 & e^{i p_{2}} & 0 \\
0 & 0 & e^{i p_{3}}
\end{array}\right)\left(\begin{array}{ccc}
A & C & -C^{*} \\
C & D^{*} & B \\
-C^{*} & B & D
\end{array}\right)\left(\begin{array}{ccc}
e^{i p_{1}} & 0 & 0 \\
0 & e^{i p_{2}} & 0 \\
0 & 0 & e^{i p_{3}}
\end{array}\right),
$$

where the phases $p_{i}$ are arbitrary.

All neutrino mass matrices which can be written in the above form, will predict $\delta= \pm \pi / 2, \theta_{23}=\pi / 4$ and all the eigen-masses are real. One can choose some particular values for $p_{i}$ to obtain forms of $m_{\nu}$ for convenience of analysis. For example the "-" sign for the " 13 " and " 31 " entries can be removed by choosing $p_{1}=p_{2}=0$ and $p_{3}=\pi$, the resultant matrix can be written in a more familiar forms

$$
m_{\nu}=\left(\begin{array}{ccc}
A & C & C^{*} \\
C & D^{*} & \tilde{B} \\
C^{*} & \tilde{B} & D
\end{array}\right)
$$

where $\tilde{B}=-B$.

\section{Theoretical model building with $\theta_{23}=\pi / 4$ and $\delta=-\pi / 2$}

The simplicity of the mass matrix discussed in the previous section may serve as a good starting point to understand the possible underlying theory. If this has something to do with reality, one should not stay at the pure phenomenological level for analysis, but go further to study whether there are theoretical models which can obtained such a neutrino mass matrix in some consistent way. Several attempts for model buildings have been made. It has been shown in ref. ${ }^{16}$ by Grimus and Lavoura that the mass matrix discussed in previous section is symmetric under a transformation of $e \rightarrow e, \mu-\tau$ exchange with a $\mathrm{CP}$ conjugation. We will refer this as the Grimus-Lavoura symmetry (GLS). In this talk we start with a simple model proposed earlier based on $A_{4}$ symmetry $^{17}$ to realize the tri-bimaximal neutrino mixing, and then modify it to allow a non-zero $\theta_{13}$ to find the conditions for having the GLS limit for neutrino mass matrix with $\delta=-\pi / 2$ and $\theta_{23}=\pi / 4$ and how modifications may occur by explicit model studies. ${ }^{14}$

Before discussing specific models, let us comment on some general properties of $A_{4}$ models which can produce $\theta_{23}=\pi / 4$ and $\delta=-\pi / 2$. One of the common features of $A_{4}$ model building is that the charged lepton mass matrix $M_{l}$ is diagonalized 
from left (rotation on left-handed charged leptons) by the characteristic matrix $U_{l}$ for $A_{4}$ symmetry model buildings, ${ }^{18}$

$$
M_{l}=U_{l} \hat{m}_{l} U_{r}, \quad U_{l}=\frac{1}{\sqrt{3}}\left(\begin{array}{lll}
1 & 1 & 1 \\
1 & \omega & \omega^{2} \\
1 & \omega^{2} & \omega
\end{array}\right),
$$

where $\omega=\exp (i 2 \pi / 3)$ and $\omega^{2}=\exp (i 4 \pi / 3)$ are the basic C-G coefficients of the $A_{4}$ group. $U_{r}$ is a unitary matrix, but does not play a role in determining $V_{P M N S}$.

If neutrinos are Majorana particles, the most general mass matrix is of the form

$$
M_{\nu}=\left(\begin{array}{lll}
w_{1} & x & y \\
x & w_{2} & z \\
y & z & w_{3}
\end{array}\right)
$$

which can be diagonlized by unitary matrix $V_{\nu}, M_{\nu}=V_{\nu} \hat{m}_{\nu} V_{\nu}^{T}$.

The mixing matrix $V_{P M N S}$ is given by

$$
V_{P M N S}=U_{l}^{\dagger} V_{\nu}
$$

In the basis where charged lepton is digonalized, the neutrino mass matrix is of the form given by eq.(8) with ${ }^{15,19}$

$$
\begin{aligned}
& a=\frac{1}{3}\left(w_{1}+w_{2}+w_{3}+2(x+y+z)\right), \\
& d+i \gamma=\frac{1}{3}\left(w_{1}+\omega w_{2}+\omega^{2} w_{3}+2\left(\omega^{2} x+\omega y+z\right)\right), \\
& d-i \gamma=\frac{1}{3}\left(w_{1}+\omega^{2} w_{2}+\omega w_{3}+2\left(\omega x+\omega^{2} y+z\right)\right), \\
& c+i \beta=\frac{1}{3}\left(w_{1}+\omega^{2} w_{2}+\omega w_{3}-\omega x-\omega^{2} y-z\right), \\
& c-i \beta=\frac{1}{3}\left(w_{1}+\omega w_{2}+\omega^{2} w_{3}-\omega^{2} x-\omega y-z\right), \\
& b=\frac{1}{3}\left(w_{1}+w_{2}+w_{3}-(x+y+z)\right) .
\end{aligned}
$$

If all parameters in the set $P=\left(w_{i}, x, y, z\right)$ are real, the mass matrix is of the form given by eq.(14) which predicts $\delta= \pm \pi / 2$ and $\theta_{23}=\pi / 4$. Therefore in $A_{4}$ model building for neutrino mixing with $\delta= \pm \pi / 2$ and $\theta_{23}=\pi / 4$, it is essential to make sure that $U_{l}$ is of the form given by eq.(15). Note that in this case since the parameters in set $P$ are all real, the complexity of the mixing matrix is purely due to the appearance of $\omega$ and $\omega^{2}$ coming from $A_{4}$ group Clebsh-Gordan coefficients. This is an interesting case where $\mathrm{CP}$ violation solely come from complexity of relevant $\mathrm{C}-\mathrm{G}$ coefficients, intrinsic $\mathrm{CP}$ violation. If the parameters in $P$ are complex, there are more sources for $\mathrm{CP}$ violation and the model does not predict $\delta= \pm \pi / 2$ and $\theta_{23}=\pi / 4$ automatically. This points a way to modify the predictions to fit data should future experiments will find $\delta$ and $\theta_{23}$ to be deviate significantly from $-\pi / 2$ and $\pi / 4$. 
We now discuss a model to realize the above idea. In this model $A_{4}$ is serving as a family symmetry. ${ }^{17}$ The Higgs sector is enlarged to have three Higgs fields, $\Phi=\left(\Phi_{1}, \Phi_{2}, \Phi_{3}\right)$ (SM doublet), $\phi$ (SM doublet) and $\chi=\left(\chi_{1}, \chi_{2}, \chi_{3}\right)$ (SM singlet). Under the $A_{4}, \Phi$ and $\chi$ both transform as 3 , and $\phi$ as 1 . Three right-handed SM singlet neutrinos $\nu_{R}=\left(\nu_{R}^{1}, \nu_{R}^{2}, \nu_{R}^{3}\right)$ are introduced allowing seesaw mechanism to be in effective. The standard left-handed leptons $l_{L}=\left(l_{L}^{1}, l_{L}^{2}, l_{L}^{3}\right)$, and standard right-handed charged leptons $\left(l_{R}^{1}, l_{R}^{2}, l_{R}^{3}\right)$, and $\nu_{R}$ transform as a $3,\left(1,1^{\prime \prime}, 1^{\prime}\right)$ and 3 , respectively. The Lagrangian responsible for the lepton mass matrix is

$$
\begin{aligned}
L & =\lambda_{e}\left(\bar{l}_{L} \tilde{\Phi}\right)_{1} l_{R}^{1}+\lambda_{\mu}\left(\bar{l}_{L} \tilde{\Phi}\right)_{1^{\prime}} l_{R}^{2}+\lambda_{\tau}\left(\bar{l}_{L} \tilde{\Phi}\right)_{1^{\prime \prime}} l_{R}^{3}+H . C . \\
& +\lambda_{\nu}\left(\bar{l}_{L} \nu_{R}\right)_{1} \phi+m\left(\bar{\nu}_{R} \nu_{R}^{C}\right)_{1}+\lambda_{\chi}\left(\bar{\nu}_{R} \nu_{R}^{C}\right)_{3} \chi,
\end{aligned}
$$

where

$$
\begin{aligned}
& \left(\bar{l}_{L} \tilde{\Phi}\right)_{1} l_{R}^{1}=\left(\bar{l}_{L} \tilde{\Phi}_{1}+\bar{l}_{L}^{2} \Phi_{2}+\bar{l}_{L}^{3} \tilde{\Phi}_{3}\right) l_{R}^{1}, \\
& \left(\bar{l}_{L} \tilde{\Phi}\right)_{1^{\prime}} l_{R}^{1}=\left(\bar{l}_{L}^{1} \tilde{\Phi}_{1}+\omega \bar{l}_{L}^{2} \Phi_{2}+\omega^{2} \bar{l}_{L}^{3} \tilde{\Phi}_{3}\right) l_{R}^{2}, \\
& \left(\bar{l}_{L} \tilde{\Phi}\right)_{1^{\prime \prime}} l_{R}^{1}=\left(\bar{l}_{L}^{1} \tilde{\Phi}_{1}+\omega^{2} \bar{l}_{L}^{2} \Phi_{2}+\omega \bar{l}_{L}^{3} \tilde{\Phi}_{3}\right) l_{R}^{3} .
\end{aligned}
$$

If the vev structure is of the form $\left.\left\langle\Phi_{1,2,3}\right\rangle=v_{\Phi},\left\langle\chi_{1,3}\right\rangle=0,<\chi_{2}\right\rangle=v_{\chi}$, and $\langle\phi\rangle=v_{\phi}$, one would obtain the charged lepton mass term as

$$
\left(\bar{l}_{L}^{1} \bar{l}_{L}^{2} \bar{l}_{L}^{3}\right) U_{l}\left(\begin{array}{lll}
\sqrt{3} \lambda_{e} v_{\Phi} & 0 & 0 \\
0 & \sqrt{3} \lambda_{\mu} v_{\Phi} & 0 \\
0 & 0 & \sqrt{3} \lambda_{\tau} v_{\Phi}
\end{array}\right)\left(\begin{array}{l}
l_{R}^{1} \\
l_{R}^{2} \\
l_{R}^{3}
\end{array}\right), \quad U_{l}=\frac{1}{\sqrt{3}}\left(\begin{array}{lll}
1 & 1 & 1 \\
1 & \omega & \omega^{2} \\
1 & \omega^{2} & \omega
\end{array}\right) .
$$

This way one obtains the desired $U_{l}$ matrix.

The neutrino mass matrix has the seesaw form with

$$
M=\left(\begin{array}{ll}
0 & M_{D} \\
M_{D}^{T} & M_{R}
\end{array}\right), \quad M_{R}=\left(\begin{array}{lll}
m & 0 & m_{\chi} \\
0 & m & 0 \\
m_{\chi} & 0 & m
\end{array}\right)
$$

where $M_{D}=\operatorname{Diag}(1,1,1) \lambda_{\nu} v_{\phi}$, and $m_{\chi}=\lambda_{\chi} v_{\chi}$. From this one obtains the light neutrino mass matrix $M_{\nu}$ of the form given by

$$
M_{\nu}=-M_{D} M_{R}^{-1} M_{D}=\left(\begin{array}{ccc}
w & 0 & x \\
0 & y & 0 \\
x & 0 & z
\end{array}\right)
$$

where $w=z=-\left(\lambda_{\nu} v_{\phi}\right)^{2} m /\left(m^{2}-m_{\chi}^{2}\right), x=\left(\lambda_{\nu} v_{\phi}\right)^{2} m_{\chi} /\left(m^{2}-m_{\chi}^{2}\right)$ and $y=$ $-\left(\lambda_{\nu} v_{\phi}\right)^{2} / m$.

The above model leads to the tri-bimaximal mixing which predicts $\theta_{13}=0$. It had been the focus of $A_{4}$ symmetry studies for a few years. ${ }^{17,20-22}$ But it is now ruled out because a non-zero $\theta_{13}$ has been measured. In this scheme, in order to obtain the tri-bimaximal mixing, the neutrino mass matrix with " 11 " and " 33 " 
entries to be equal is crucial. It has been pointed out ${ }^{17,21}$ that a more natural form of vev structure will lead to the " 33 " entry in the neutrino mass matrix to be deviate from the " 11 " entry which leads to a non-zero $\theta_{13}$. To achieve this, for our purpose here, we will introduce two scalars $S_{1^{\prime}}$ and $S_{1^{\prime \prime}}$ which are SM singlet but transform as $1^{\prime}$ and $1^{\prime \prime}$ under $A_{4}$. This results in two new terms for $M_{R}$ in the Lagrangian ${ }^{14}$

$$
Y_{S^{\prime}}\left(\bar{\nu}_{R} \nu_{R}^{C}\right)_{1^{\prime \prime}} S_{1^{\prime}}+Y_{S^{\prime \prime}}\left(\bar{\nu}_{R} \nu_{R}^{C}\right)_{1^{\prime}} S_{1^{\prime \prime}}+\text { H.C. }
$$

After $S_{1^{\prime}, 1^{\prime \prime}}$ develops a non-zero vev, $v_{S^{\prime}, S^{\prime \prime}}$, we have

$$
M_{R}=\left(\begin{array}{lll}
m_{1} & 0 & m_{\chi} \\
0 & m_{2} & 0 \\
m_{\chi} & 0 & m_{3}
\end{array}\right),
$$

where $m_{1}=m+Y_{S^{\prime}} v_{S^{\prime}}+Y_{S^{\prime \prime}} v_{S^{\prime \prime}}, m_{2}=m+\omega^{2} Y_{S^{\prime}} v_{S^{\prime}}+\omega Y_{S^{\prime \prime}} v_{S^{\prime \prime}}$ and $m_{3}=$ $m+\omega Y_{S^{\prime}} v_{S^{\prime}}+\omega^{2} Y_{S^{\prime \prime}} v_{S^{\prime \prime}}$. The resulting light neutrino mass matrix $M_{\nu}$ no longer has $w=z$, but has

$$
w=-\lambda_{\nu}^{2} v_{\phi}^{2} m_{3} /\left(m_{1} m_{2}-m_{\chi}^{2}\right), \quad z=-\lambda_{\nu}^{2} m_{1} /\left(m_{1} m_{3}-m_{\chi}^{2}\right),
$$

and $x$ and $y$ are changed to

$$
x=\lambda_{\nu}^{2} v_{\phi}^{2} m_{\chi} /\left(m_{1} m_{3}-m_{\chi}^{2}\right), \quad y=-\lambda_{\nu}^{2} v_{\phi}^{2} / m_{2} .
$$

In the basis where the charged lepton mass matrix is diagonalized, the neutrino mass matrix becomes

$$
\begin{aligned}
m_{\nu} & =U_{l}^{\dagger} M_{\nu} U_{l}^{*} \\
& =\frac{1}{3}\left(\begin{array}{ccc}
w+2 x+y+z & w-\omega^{2} x+\omega^{2} y+\omega z & w-\omega x+\omega y+\omega^{2} z \\
w-\omega^{2} x+\omega^{2} y+\omega z & w+2 \omega x+\omega y+\omega^{2} z & w-x+y+z \\
w-\omega x+\omega y+\omega^{2} z & w-x+y+z & w+2 \omega^{2} x+\omega^{2} y+\omega z
\end{array}\right) .
\end{aligned}
$$

The above $m_{\nu}$ can be transformed into the form in eq.(8) by redefine righthanded charged leptons. The parameters in the set $P_{A 4}:\{w, x, y, z\}$ are in general complex which will not always have $\delta=-\pi / 2$ and $\theta_{23}=\pi / 4$. One needs to work in the GLS limit which can be realized if the parameters in the set $P_{A 4}$ are all real. In this case the complexity of the mass matrix is purely due to the $A_{4}$ group theoretical C-G coefficients $\omega$ and $\omega^{2}$. This is a case where $\mathrm{CP}$ violation is caused by $\mathrm{C}-\mathrm{G}$ coefficients providing a concrete example of intrinsic $\mathrm{CP}$ violation.

Before we analysis the general features of the neutrino mass matrix with complex parameters in the set $P_{A_{4}}$, we would like to analysis the constraints on the model parameters to have the GLS limit, that is, to have $w, x, y, z$ to be real. The complexity of the parameters can appear in the Yukawa couplings, in the vevs, and also in places where $\omega^{i}$ appear in $m_{i}$. To make the Yukawa couplings and scalar vevs 
real, one can require the model Lagrangian to satisfy a generalized CP symmetry under which

$$
\begin{aligned}
& \left(l_{L}^{1}, \quad l_{L}^{2}, l_{L}^{3}\right) \rightarrow\left(\left(l_{L}^{1}\right)^{C P}, \quad\left(l_{L}^{3}\right)^{C P},\left(l_{L}^{2}\right)^{C P}\right), \\
& \left(\nu_{R}^{1}, \nu_{R}^{2}, \nu_{R}^{3}\right) \rightarrow\left(\left(\nu_{R}^{1}\right)^{C P}, \quad\left(\nu_{R}^{3}\right)^{C P}, \quad\left(\nu_{R}^{2}\right)^{C P}\right), \\
& \left(\Phi_{1}, \Phi_{2}, \Phi_{3}\right) \rightarrow\left(\Phi_{1}^{\dagger}, \Phi_{3}^{\dagger}, \Phi_{2}^{\dagger}\right), \\
& \left(\chi_{1}, \chi_{2}, \chi_{3}\right) \rightarrow\left(\chi_{1}^{\dagger}, \quad \chi_{3}^{\dagger}, \chi_{2}^{\dagger}\right), \\
& \left(S_{1^{\prime}}, \quad S_{1^{\prime \prime}}\right) \rightarrow\left(S_{1^{\prime}}^{\dagger}, S_{1^{\prime \prime}}^{\dagger}\right),
\end{aligned}
$$

and all other fields transform the same as those under the usual CP symmetry. Here the superscript $C P$ in the above indicates that the fields are the usual $C P$ transformed fields.

The above transformation properties will transform relevant terms into their complex conjugate ones. Requiring the Lagrangian to be invariant under the above transformation dictates the Yukawa couplings to be real. The same requirement will dictates the scalar potential to forbid spontaneous CP violation and vevs to be real. One, however, notices that the parameters $m_{2,3}$ are in general complex even if the Yukawa couplings and the vevs of the scalar fields are made real because of the appearance of $\omega^{i}$. To make them real to reach GLS limit, it is therefore required that

$$
\operatorname{Im}\left(\omega^{2} Y_{S^{\prime}} v_{S^{\prime}}+\omega Y_{S^{\prime \prime}} v_{S^{\prime \prime}}\right)=\operatorname{Im}\left(\omega Y_{S^{\prime}} v_{S^{\prime}}+\omega^{2} Y_{S^{\prime \prime}} v_{S^{\prime \prime}}\right)=0 .
$$

The above can be achieved by the absent of the scalar fields $S^{\prime},{ }^{\prime \prime}$ in the theory or set $Y_{S^{\prime}} v_{S^{\prime}}=Y_{S^{\prime \prime}} v_{S^{\prime \prime}}$. If the vev structure of $\chi$ is fixed as given previously, absence of $S^{\prime}$," will not have a phenomenologically acceptable mass matrix. Therefore, we will take the later possibility as example of GLS limit case to show some detailed features.

In this case $M_{\nu}$ can be diagonalized by $V_{\nu}$ as the following

$$
M_{\nu}=V_{\nu} \hat{m}_{\nu} V_{\nu}^{T}, \quad V_{\nu}=\left(\begin{array}{ccc}
c & 0 & -s \\
0 & 1 & 0 \\
s & 0 & c
\end{array}\right)
$$

where $s=\sin \theta$ and $c=\cos \theta$. One obtains the mixing matrix to be

$$
V_{P M N S}=U_{l}^{\dagger} V_{\nu}=\frac{1}{\sqrt{3}}\left(\begin{array}{ccc}
c+s & 1 & c-s \\
c+\omega s & \omega^{2} & \omega c-s \\
c+\omega^{2} s & \omega & \omega^{2} c-s
\end{array}\right),
$$

Normalizing the above mixing matrix to the standard parametrization in eq.(3), one obtains ${ }^{14,15}$

$$
s_{12}=\frac{1}{\sqrt{2(1+c s)}}, s_{23}=\frac{1}{\sqrt{2}}, \quad s_{13}=\frac{(1-2 c s)^{1 / 2}}{\sqrt{3}} .
$$

Here we have normalized $c_{i j}$ and $s_{i j}$ to be all positive. The neutrino eigen-masses are all real, but in general they can take positive or negative values depending on 
the values of $w, x, y$ and $z$. Note that the absolute values of elements in the second column of $V_{P M N S}$ are all $1 / \sqrt{3}$.

We now find the conditions for predicting $\delta=-\pi / 2$ and $\delta=+\pi / 2$. An easy way of doing this is to study the Jarlskog invariant quantity ${ }^{23} \mathrm{~J}=\operatorname{Im}\left(V_{e 1} V_{e 2}^{*} V_{\mu 1}^{*} V_{\mu 2}\right)$. Eqs.(3) and (32) give gi, $15^{15}$

$$
J=c_{13}^{2} s_{12} c_{12} s_{23} c_{23} s_{13} \sin \delta=-\frac{1}{6 \sqrt{3}}\left(c^{2}-s^{2}\right),
$$

which leads to

$$
\delta=\frac{\pi}{2} \times \begin{cases}-1, & \text { if } c^{2}>s^{2}, \\ +1, & \text { if } s^{2}>c^{2} .\end{cases}
$$

Note that $J$ is not zero implying CP violation which is caused by the complexity of C-G coefficients. Eq. (32) can be transformed into the standard parameterization by multiplying the $V_{P M N S}$ on the right and left by diagonal matrices $P_{r}=\operatorname{diag}(1,1, i)$ and $P_{l}=\operatorname{diag}\left(1,\left(\omega^{2} c-s\right) /\left|\omega^{2} c-s\right|,(\omega c-s) /|\omega c-s|\right)$, respectively. $P_{l}$ does not have physical effect because it can be absorbed by redefinition of right-handed charged leptons. The physical effects of $P_{r}$ is to change the sign of $m_{3}$.

\section{Discussions and conclusions}

Let us now compare experimental data with the model predictions for the mixing angles and CP violating phase. There are several global fits of neutrino data. ${ }^{4}$ The latest fit gives the central values, $1 \sigma$ errors and the $2 \sigma$ ranges as the following ${ }^{24}$

$$
\begin{aligned}
& \delta / \pi \\
& \mathrm{NH} \quad 1.41_{-0.44}^{+0.55} \\
& 2 \sigma \text { region } \quad 0.0 \sim 2.0 \\
& \text { IH } \quad 1.48 \pm 0.31 \\
& s_{12}^{2} \quad s_{13}^{2} / 10^{-2} \\
& s_{23}^{2} \\
& 0.323 \pm 0.0162 .26 \pm 0.12 \quad 0.567_{-0.124}^{+0.032} \\
& 0.292 \sim 0.3572 .02 \sim 2.500 .414 \sim 0.623 \\
& 0.323 \pm 0.0162 .29 \pm 0.12 \quad 0.573_{-0.039}^{+0.025} \\
& 2 \sigma \text { region } 0.00 \sim 0.09 \& 0.86 \sim 2.00 .292 \sim 0.3572 .05 \sim 2.520 .435 \sim 0.621
\end{aligned}
$$

Here $N H$ and $I H$ indicate neutrino mass hierarchy patterns of normal hierarchy and inverted hierarchy, respectively.

In the model discussed earlier, adjusting the values, $w, x, y$ and $z$, both $\mathrm{NH}$ and IH mass patterns can be obtained. There is strong hint that the Dirac phase should be close to $3 \pi / 2$ (or equivalently $-\pi / 2$ ). Therefore one should take the parameter space so that $c^{2}>s^{2}$. The value $-\pi / 2$ predicted in the model is in agreement with $\mathrm{IH}$ within $1 \sigma$ range. Although for $\mathrm{NH}$ case $\delta$ is outside of $1 \sigma$ range, there no problem with $2 \sigma$ range. For $s_{23}$, the model predicts $s_{23}^{2}=0.5$. This value is outside of $1 \sigma$ range for both the $\mathrm{NH}$ and $\mathrm{IH}$ cases. However, they are, again, in agreement with data within $2 \sigma$.

In the model $s_{13}=(1-2 c s)^{1 / 2} / \sqrt{3}$ is not predicted. But one can use information from $s_{13}$ to fix $c s=0.497 \pm 0.018$ to predict $s_{12}^{2}=0.334 \pm 0.004$ for both NH and IH 
cases. This is in agreement with data within $1 \sigma$. Note that $V_{e 2}^{2}=\left(s_{12} c_{13}\right)^{2}=1 / 3$. It agrees with data within $1 \sigma$. It is remarkable that neutrino mixing matrix in this model with just one free parameter can be in reasonable agreement with data. This may be a hint that it is the form for mixing matrix, at least as the lowest order approximation, that a underlying theory is producing.

If $w, x, y$ and $z$ are allowed to be complex, the GLS is explicitly broken, there are modifications to the mixing angles. There is additional source for $\mathrm{CP}$ violation other than the intrinsic one from complexity of $\mathrm{C}-\mathrm{G}$ coefficient, and also the mixing angles will be modified. The eigen-masses will contain Majorana phases. Detailed analysis of how to diagonalize the mass matrix has been discussed in Ref.. ${ }^{21}$ In general this model does not always predicts $\delta= \pm \pi / 2$ and $\theta_{23}=\pi / 4$. The mixing matrix can be, in general, written as

$$
V_{P M N S}=U_{l}^{\dagger} V_{\rho} V_{\nu}=\frac{1}{\sqrt{3}}\left(\begin{array}{ccc}
c+s e^{i \rho} & 1 & c e^{i \rho}-s \\
c+\omega s e^{i \rho} & \omega^{2} & \omega c e^{i \rho}-s \\
c+\omega^{2} s e^{i \rho} & \omega & \omega^{2} c e^{i \rho}-s
\end{array}\right),
$$

where $V_{\rho}$ is a diagonal matrix $\operatorname{diag}\left(1,1, e^{i \rho}\right)$ with $\tan \rho=\operatorname{Im}\left(x w^{*}+x^{*} z\right) / \operatorname{Re}\left(x w^{*}+\right.$ $\left.x^{*} z\right)$. It is interesting that the phase $\rho$ does not show up in $J$ which is still $-\left(c^{2}-\right.$ $\left.s^{2}\right) / 6 \sqrt{3}$. This implies that CP violation related to neutrino oscillation is still purely due to intrinsic CP violation. The mixing angles and the Dirac phase $\delta$ are all modified with

$$
\begin{aligned}
& s_{12}=\frac{1}{\sqrt{2}(1+c s \cos \rho)^{1 / 2}}, \\
& s_{23}=\frac{(1+c s \cos \rho+\sqrt{3} c s \sin \rho)^{1 / 2}}{\sqrt{2}(1+c s \cos \rho)^{\frac{1}{2}}}, \\
& s_{13}=\frac{(1-2 c s \cos \rho)^{1 / 2}}{\sqrt{3}},
\end{aligned}
$$

and

$$
\sin \delta=\left(1+\frac{4 c^{2} s^{2} \sin ^{2} \rho}{\left.\left(c^{2}-s^{2}\right)^{2}\right)}\right)^{-1 / 2}\left(1-\frac{3 c^{2} s^{2} \sin ^{2} \rho}{(1+c s \cos \rho)^{2}}\right)^{-1 / 2} \times\left\{\begin{array}{ll}
-1, & \text { if } c^{2}>s^{2} \\
+1, & \text { if } s^{2}>c^{2}
\end{array} .\right.
$$

In this case, the new parameter $\rho$ can be used to improve agreement of the model with data. In both $\mathrm{NH}$ and $\mathrm{IH}$ cases, $\delta$ and $s_{23}$ can be brought into agreement with data at $1 \sigma$ level. To see how this can be done, as an example, we take the largest value of $c s$ so that $s_{13}$ takes its lower $1 \sigma$ allowed value, and then varying $\cos \rho$ to obtain the upper $1 \sigma$ allowed value. This fixes $c s$ and $\cos \rho$ to be 0.468 and 0.992 , respectively. With these values, $s_{23}$ and $\delta$ are determined to: 0.534 and $1.426 \pi$, respectively. These values are in agreement with data at $1 \sigma$ level. When more precise experimental data become available, the model with complex model parameters can be distinguished from that with the parameters are all real and other models. 
In conclusion we have shown that neutrino mass matrix reconstructed with $\delta=$ $-\pi / 2$ and $\theta_{23}=\pi / 4$ has several interesting properties. We find that a theoretical model based on the $A_{4}$ symmetry naturally realize the GLS limit and predicts such a neutrino mixing pattern together with the prediction $\left|V_{e 2}\right|=1 / \sqrt{3}$. In this model, $\mathrm{CP}$ violation can be solely come from the complex group theoretical C-G coefficients if the neutrino Majorana phases are zero or $\pi$. This model fits experimental data very well and can be taken as the lowest order neutrino mass matrix for future theoretical model buildings. If there are additional source of CP violation other than those intrinsically existed in the $\mathrm{C}-\mathrm{G}$ coefficients, the $\mathrm{CP}$ violating phase $\delta$ and the mixing angle $\theta_{23}$ can be away from $-\pi / 2$ and $\pi / 4$. The models discussed can fit data within $1 \sigma$. Future improved experimental data will be able to further test the model and provide more hints for the underlying theory of neutrino mixing.

I thank Guan-Nan Li for collaboration on related studies. The work was supported in part by MOE Academic Excellent Program (Grant No: 102R891505) and MOST of ROC, and in part by NSFC(Grant No:11175115) and Shanghai Science and Technology Commission (Grant No: 11DZ2260700) of PRC.

\section{References}

1. Y. Fukuda et al., (Super-Kamiokande collaboration), Phys. Rev. Lett. 81, 1562 (1998).

2. Q. R. Ahmad et al (SNO collaboration), Phys. Rev. Lett. 87, 071301(2001); Phys. Rev. Lett. 89, 011301(2002).

3. Z. Maki, M. Nakagawa and S. Sakata, Prog. Theor. Phys. 28, 870(1962); B. Pontecorvo, Sov. Phys. JTEP 26, 984(1968).

4. K.A. Olive et al. (Particle Data Group), Chin. Phys. C, 38, 090001 (2014).

5. L.L. Chau and W.Y. Keung, Phys. Rev. Lett. 53, 1802(1984).

6. See for example: A. J. Davies and Xiao-Gang He, Phys. Rev. D46, 3208(1992).

7. V. D. Barger, S. Pakvasa, T. J. Weiler and K. Whisnant, Phys. Lett. B 437, 107 (1998) doi:10.1016/S0370-2693(98)00880-6 [hep-ph/9806387].

8. P. F. Harrison and W.G. Scott, Phys. Lett. B535, 163(2002); P.F. Harrison and W.G. Scott, Phys. Lett. B557, 76(2003); Z.-Z. Xing, Phys. Lett. B533, 85(2002); X. G. He and A. Zee, Phys. Lett. B 560, 87 (2003).

9. F. P. An et al. [Daya Bay Collaboration], Phys. Rev. Lett. 108, 171803 (2012) doi:10.1103/PhysRevLett.108.171803 [arXiv:1203.1669 [hep-ex]].

10. J. K. Ahn et al. [RENO Collaboration], Phys. Rev. Lett. 108, 191802 (2012) doi:10.1103/PhysRevLett.108.191802 [arXiv:1204.0626 [hep-ex]].

11. M. R. Salzgeber [T2K Collaboration], arXiv:1508.06153 [hep-ex].

12. R. Patterson [NO?A Collaboration], talk given at the Joint Experimental-Theoretical Physics Seminar, Fermi- lab, 6 August 2015.

13. M. Kobayashi and T. Maskawa, Prog. Theor. Phys. 49, 652 (1973).

14. X. G. He, Chin. J. Phys. 53, 100101 (2015) [arXiv:1504.01560 [hep-ph]].

15. G. N. Li and X. G. He, Phys. Lett. B 750, 620 (2015) [arXiv:1505.01932 [hep-ph]].

16. W. Grimus and L. Lavoura, Phys. Lett. B 579, 113 (2004).

17. X. G. He, Y. Y. Keum and R. R. Volkas, JHEP 0604, 039 (2006).

18. E. Ma and G. Rajasekaran, Phys. Rev. D64, 113012 (2001); K. S. Babu, E. Ma, and J. W. F. Valle, Phys. Lett. B552, 207 (2003).

19. E. Ma, Phys. Rev. D 92, no. 5, 051301 (2015) doi:10.1103/PhysRevD.92.051301 [arXiv:1504.02086 [hep-ph]]. 
20. G. Altarelli and F. Feruglio, Nucl. Phys. B720, 64(2005); K. S. Babu and X. G. He, hep-ph/0507217.

21. X. G. He and A. Zee, Phys. Lett. B 645, 427 (2007).

22. X. G. He, Nucl. Phys. Proc. Suppl. 168, 350 (2007).

23. C. Jarlskog, Phys. Rev. Lett. 55, 1039 (1985).

24. D. V. Forero, M. Tortola and J. W. F. Valle, Phys. Rev. D 90, no. 9, 093006 (2014). 